\title{
Un espacio personal en la web
}

A personal web space

\author{
María Elena Tosello \\ Universidad Nacional del Litoral, Argentina \\ maritosello@gmail.com
}

\author{
María Georgina Bredanini Colombo \\ Universidad Nacional del Litoral, Argentina \\ arq_bredanini@yahoo.com.ar
}

\begin{abstract}
We live constantly networked, performing multiple activities in virtual spaces, which are intertwined with physical space, shaping an augmented and symbiotic cronotopo. Considering that personal space is an area surrounding individuals that provides a framework for developing activities ¿wouldn't be necessary to count on a virtual personal space? This paper presents the basis, processes and results of a didactic experience whose purpose was to imagine and design a personal web space, representing its properties and characteristics through a transmedia narrative deployed in diverse languages and media
\end{abstract}

Keywords: Espacio personal; Narrativa transmedia; Espacio virtual; Diseño paramétrico; Diseño de videojuegos.

\section{Introducción}

La configuración del espacio deriva de la forma de adecuación del hombre a su entorno y se presenta como una estructura total que incluye objetos físicos, psíquicos, sociales y culturales, distribuidos en diferentes niveles que se influyen mutuamente (Norberg-Schulz, 1975), conformando un ambiente complejo y dinámico compuesto por las cosas, la casa, la ciudad, el paisaje y los espacios virtuales.

La casa por ejemplo, representa la necesidad de estar situados. Es el lugar central de la existencia humana, allí el hombre aprende a compartir el mundo. Es el lugar de donde parte y al que regresa. La casa es el primer universo, donde en la más interminable de las dialécticas, el ser amparado se sensibiliza, vive la casa en su realidad y en su virtualidad, con pensamientos, memorias y sueños (Bachelard, 1975). La casa expresa una forma de vida, expresa la estructura del habitar con todos sus aspectos físicos y psíquicos, es un sistema concretizado de actividades significativas que consta de ámbitos de diverso carácter que se afectan unos a otros. Así como algunas formas de vida privilegian las actividades sociales por sobre las privadas, así también hay casas con carácter más público.

Como el espacio es fruto de la interacción entre el hombre y el ambiente, no se puede disociar la organización del mismo de las actividades que en él se desarrollan. Dentro de las dimensiones del espacio, el "espacio personal" proporciona un marco al desenvolvimiento de las actividades. Una

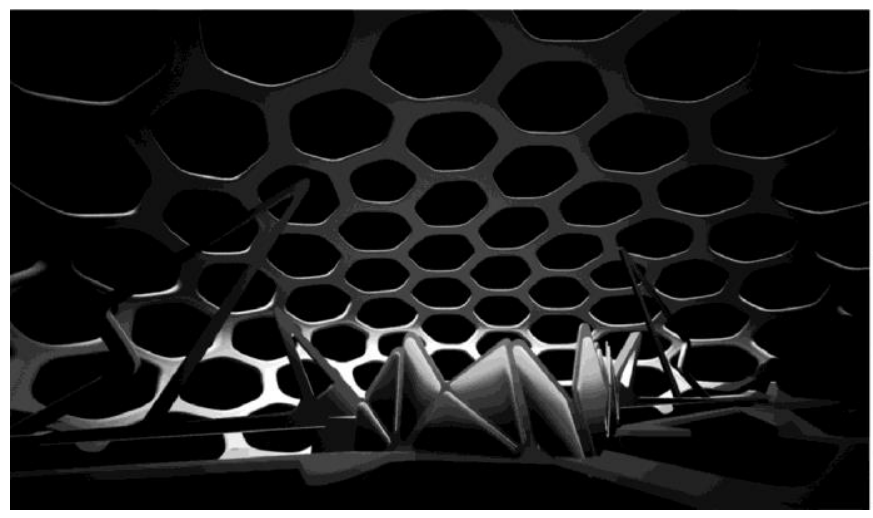

condición básica de este tipo de espacio, es un claro sentido de los límites virtuales que marcan las distancias adecuadas entre las personas (proxémica). Hall (1966) ha definido al espacio personal como una zona que rodea al individuo, en donde no puede entrar otra persona sin su autorización, es como una burbuja a su alrededor que crea un distanciamiento, un límite invisible entre la persona y los demás. Esta burbuja es variable en función a preferencias individuales, circunstancias contextuales y a la naturaleza particular de las relaciones interpersonales.

El espacio personal comprende un complejo patrón de interacciones y conductas relacionadas (Sommer, 1969), y aunque a menudo se considera circular, existen estudios que indican que éste podría presentar una forma más compleja (Holahan, 2000). Los modelos del espacio personal y de las conductas espaciales parten de la teoría general de sistemas, fundándose en la idea de que tanto los sistemas biológicos como los sociales, están compuestos por diversas variables que funcionan entrelazadas para mantener el estado de equilibrio a través del tiempo.

\section{Un espacio personal en la web}

Según Foucault, el espacio es siempre "espacio vivido". El autor sostiene que la vivencia actual del espacio, hace evidente su carácter heterogéneo y provoca el surgimiento de espacios-otros o heterotopías, que tienen la propiedad de estar en relación con todos los demás emplazamientos de una cultura (Foucault, en García Canal, 1999), afectando las experiencias humanas:
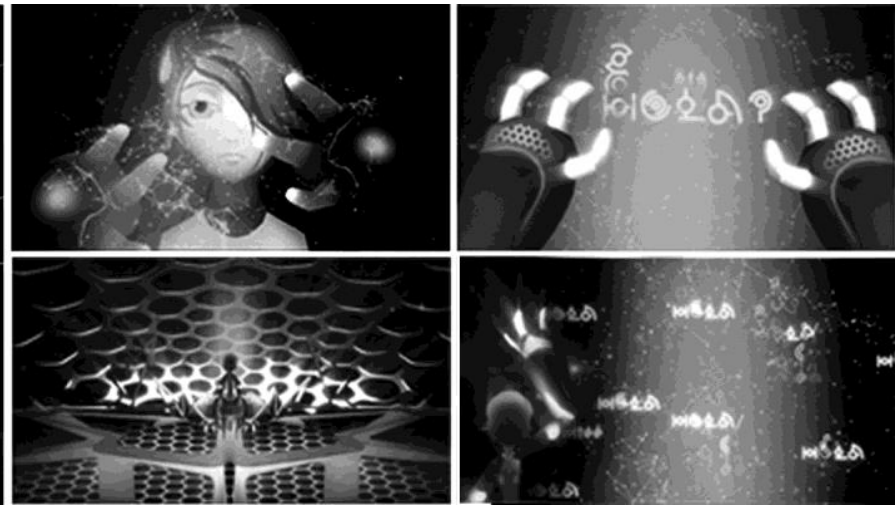

Figura 1: Modelo 3D y escenas video digital Caso 1 (Grupo Sandoval, Sariego, Scheggia, Sokolowsky). 

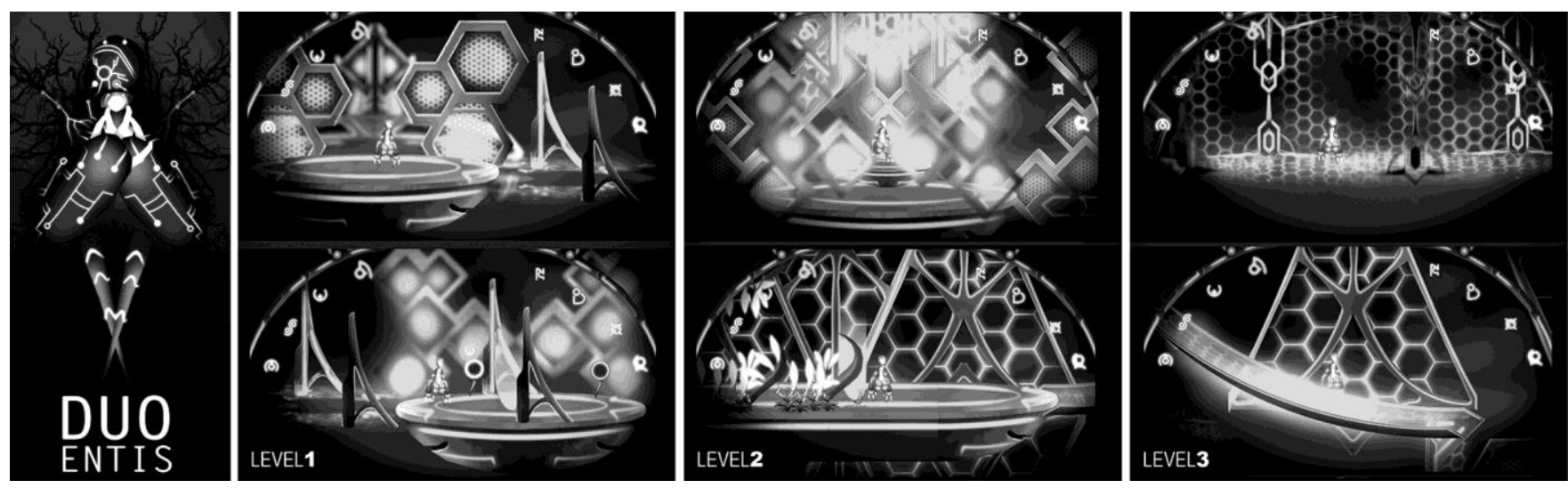

Figura 2: Hipermedio Lúdico Caso 1 (Grupo Sandoval, Sariego, Scheggia, Sokolowsky).

"Estamos en la era de la simultaneidad... de la proximidad y la lejanía... de la contigüidad y la dispersión. Nos encontramos en un momento, creo yo, en el cual el mundo ya no se experimenta tanto como una gran vía que se extiende en el tiempo, sino como una red que une puntos y que entrecruza su madeja." (Foucault, 1994: 31)

Vivimos entre-medios, constantemente conectados en red y realizando múltiples actividades en espacios virtuales, los cuales se entrelazan con el espacio físico de nuestro cuerpo configurando un cronotopo aumentado y simbiótico. Considerando que el espacio personal es la zona que nos rodea y que proporciona un marco para el desenvolvimiento de las actividades ¿no sería necesario disponer también de un espacio personal en la web?

Un espacio de apropiación personal en la web sustentado en la metáfora de la "casa", debería integrar ámbitos privados (de trabajo, lectura, esparcimiento, etc.), con espacios públicos que permitan el encuentro con otros, el intercambio dialógico y la colaboración.

Además en la virtualidad, una casa sería flexible y móvil, podría expandirse, achicarse o plegarse, ajustándose al "modo privado" o al "modo público" a partir de la definición de diferentes niveles de acceso o privacidad, y podría acoplar dinámicamente recursos y servicios. Sería una "casa-nube" que nos acompañaría y asistiría en todos los recorridos y actividades, mediante tecnologías ubicuas que integran la información al entorno móvil de las personas, facilitando los intercambios constantes.

Este trabajo presenta los fundamentos, procesos y resultados de una experiencia didáctica cuyo propósito fue imaginar y diseñar un espacio personal en la web -EPW-, representando sus características formales y sus propiedades performativas mediante una narrativa transmedia -NT- desplegada a través de diversos medios y lenguajes, que colaboran en la construcción de sentido del relato.

La NT debía aprovechar sinérgica y convergentemente las potencialidades de cada plataforma. La convergencia designa una situación en la que se integran medios de diversa naturaleza, a través de los cuales fluyen los contenidos, e implica procesos dinámicos de intersección entre diferentes sistemas (Jenkins, 2009).

Nos encontramos inmersos en una nueva ecología mediática caracterizada por la convergencia de medios, que posibilita la emergencia de nuevos modelos narrativos. Al interior de éstos, los contenidos se expanden, se retroalimentan y circulan por múltiples plataformas... Diferentes lenguajes, soportes, dispositivos y géneros, se ponen al servicio de un ecosistema de relatos convergentes (Cátedra Latinoamericana de Narrativas Transmedia, 2015).

\section{Metodología}

"Un Espacio Personal en la Web" fue la consigna de todo el primer cuatrimestre de 2016 en el Taller de Gráfica Digital de la FADU-UNL. La experiencia se abordó en grupos interdisciplinarios conformados por estudiantes del ciclo superior de las carreras de Arquitectura, Diseño en Comunicación Visual y Diseño Industrial, para realizar tres trabajos prácticos como una producción integrada, considerando la convergencia de medios de la propuesta. Todos los trabajos tenían como eje conductor, el diseño y la comunicación a través de la colaboración y la participación en los espacios físicos y virtuales del taller.

\section{Cada trabajo práctico integró un medio diferente:}

- Un video digital de 2 a 4 minutos de duración, que expresara la interpretación del grupo de las características fenomenológicas y posibles vivencias en el EPW, proyectado desde la mirada subjetiva de sus habitantes. Cada grupo además, debía elaborar un guión, un storyboard y una memoria descriptiva de la propuesta.

El video digital es el más maduro de los nuevos medios, porque su proximidad con el cine le provee de herramientas narrativas y de valores formales arraigados, y su diálogo con las artes plásticas le aporta un sólido andamiaje conceptual que permite desarrollar una estética y un conjunto de procedimientos propios.

- Un objeto tridimensional complejo que representara parte o totalidad del EPW, diseñado a partir de lógicas de modelado 
paramétrico, en base a variables dinámicas que permiten la manipulación y el control de propiedades y relaciones entre las entidades geométricas. La entrega incluía un panel impreso con la fundamentación, el proceso de diseño y los resultados obtenidos, y un GIF animado con las relaciones y evolución de los espacios propuestos.

En los procesos de diseño paramétrico, todos los actos o decisiones de diseño, interpretan y evalúan parámetros, cuyos cambios afectan al elemento geométrico y a cualquier entidad asociada o dependiente de él, posibilitando la exploración de una morfología variable que responde y se adapta a los requerimientos en forma integral y dinámica.

- Un hipermedio lúdico, que a partir de una narrativa interactiva y a través del juego propusiera experiencias que permitan vivenciar las características fenomenológicas del EPW, recurriendo a los personajes y relatos previamente definidos, y a los escenarios y objetos diseñados.

Flusser (1967) aporta la noción de homo ludens, que se suma a los de homo sapiens y homo faber, para representar una nueva distinción entre los seres humanos y otros seres vivos, entendiendo que existe una correlación entre juego y cultura, y que los principios de los juegos serían las bases de las instituciones sociales. Define al juego como un sistema compuesto por elementos combinables de acuerdo con reglas, donde el conjunto de elementos son el "repertorio del juego", el conjunto de reglas, la "estructura del juego", y la totalidad de combinaciones posibles, el "universo del juego".

\section{Resultados}

Se presentan tres casos, que fueron seleccionados por plantear diferentes estrategias de abordaje del problema:

\section{Caso 1: Grupo Sandoval, Sariego, Scheggia, Sokolowsky}

Las principales premisas de diseño que el grupo se planteó al diseñar su propuesta de espacio personal en la web, fueron: lograr una narración interactiva no lineal; incluir contenido abierto; posibilitar que el propio usuario genere la estructura de contenidos con su navegación a través de una interfaz variable, otorgándole más libertad a la hora de observar y modificar, y convirtiéndolo en lo que Toffler define como prosumidor (Scolari, 2013).

El grupo se propuso generar un relato rizomático, donde existan distintos puntos de interés y se den múltiples interacciones, entradas y salidas. En este espacio interactivo se busca lograr un vínculo entre forma, metáfora y estructura, creando una relación entre lo hipermedial y lo contributorio. Hipermedial porque desde cualquier lugar se puede ir a otro en todo momento, y contributorio porque el usuario puede añadir sucesos que luego serán opciones que los siguientes usuarios podrán elegir.

Dentro de la narración interactiva de la propuesta, se optó por utilizar un estilo de aventura en mundos y relatos de varios jugadores. Diversas unidades narrativas son articuladas en una red, donde el usuario va descubriendo a medida que explora el espacio virtual y encuentra la información necesaria, y por existir más de un participante, ofrece la posibilidad de que usuarios distantes interactúen en un mismo espacio virtual. Esta situación ha sido representada particularmente en el guión del video digital, donde el protagonista busca interactuar con otros usuarios conectados a la red (Figura 1).

La participación de otros usuarios dentro del espacio personal en la web, se propone con el sentido de generar un proceso de transformación cultural que involucre distintos medios de comunicación: una cultura participante, que no sólo consume sino que también crea, aludiendo a la convergencia mediática que plantea Jenkins (2009).

La propuesta estética del espacio, lograda a través del diseño paramétrico, atraviesa todos los medios narrativos planteados por el grupo, y constituye el principal anclaje para el desarrollo del hipermedio lúdico. La idea del juego, denominado "DUO ENTIS", está basada en cómo se manifiestan los diferentes estados emocionales del usuario al interactuar en su espacio personal en la web. El usuario va a participar y manifestarse en este espacio, generando una construcción de su "Yo" virtual o alter ego, cargado de estados emocionales, ya sean positivos o negativos. A lo largo del juego, el personaje deambulará por los diferentes escenarios, dejando su huella digital en el mundo virtual, evitando que su opuesto o "alter ego negativo" distorsione el entorno (Figura 2).

\section{Caso 2: Grupo Malato, Menéndez, Navarro, Schmidt}

De acuerdo a lo expresado por el grupo, la idea motivadora de la propuesta fue representar al usuario del espacio personal en la web como un "reflejo", que habita un espacio digital de carácter abstracto. Imaginan al EPW como un mundo paralelo, donde es posible vivir experiencias en diferentes ámbitos y escenarios, navegando como una figura geométrica por páginas, sitios y redes, a las que se accede diariamente con distintos objetivos (estudio, trabajo, diversión, etc.). El espejo, motivador de la idea, ha sido un
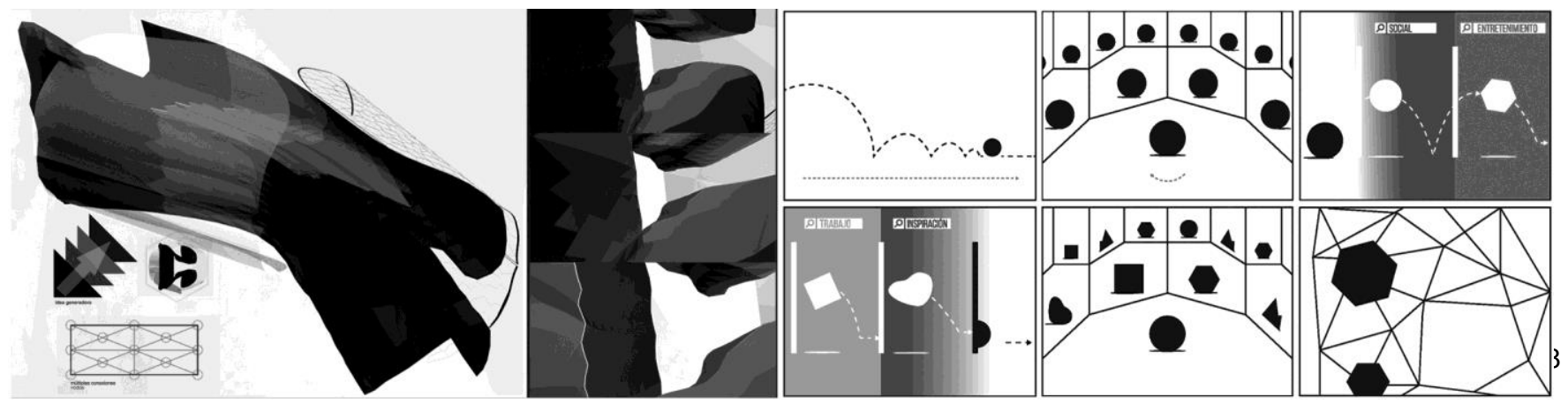

Figura 3: Modelo 3D y escenas del video digital Caso 2 (Grupo Malato, Menéndez, Navarro, Schmidt). 

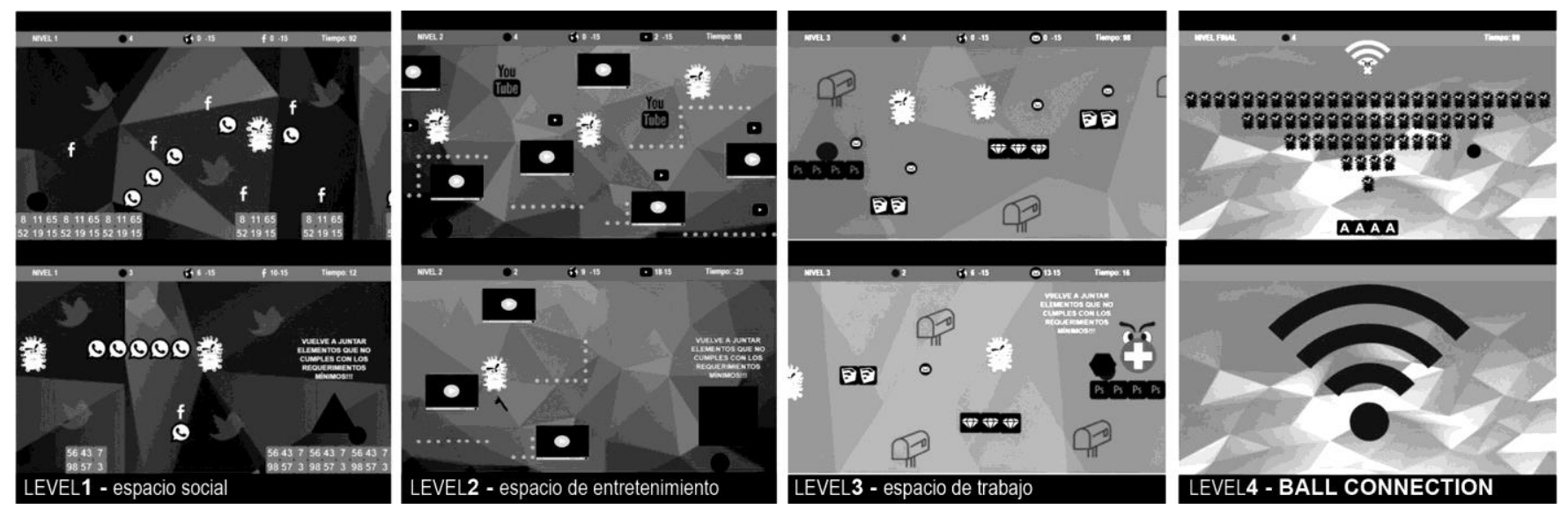

Figura 4: Hipermedio Lúdico Caso 2 (Grupo Malato, Menéndez, Navarro, Schmidt).

elemento destacado por el grupo por su amplia variedad de connotaciones, y por interpretar una analogía entre lo que ocurre con nuestra realidad y la web, y la historia de "Alicia a través del espejo" de Lewis Carroll, en la cual el personaje atraviesa un espejo pasando a otro mundo, donde vive diferentes aventuras y convive con diversos personajes. A su vez, vinculan esta idea con la relación de co-implicación entre el sujeto y el mundo que plantea Merleau-Ponty (1957), percibiendo los espacios y construyendo una realidad en su conciencia.

En la narrativa propuesta por el grupo, el protagonista (el "usuario"), es un personaje abstracto que se encontrará a sí mismo, rodeado por sus propios reflejos. Descubre que al atravesar un espejo, se encuentra con diferentes espacios en los que se integran sitios web, redes sociales, servicios, herramientas, etc. El protagonista irá mutando dependiendo del espacio en el que se encuentre, es decir, según el espacio cambian sus características, transformándose en un cuadrado, un triángulo, un hexágono o una forma irregular; en tanto cambian también las características de los espacios, sus colores, su música, su configuración espacial, etc. Esta situación pretende representar que una persona puede adoptar diferentes perfiles, según el espacio digital en el que se encuentre, de allí la idea original de representar el usuario como un reflejo, siendo éste el que cambia y muta según el lugar y la actividad que esté realizando. Al finalizar el video, puede observarse que el ámbito donde se encuentra el usuario es tan sólo un nodo dentro de un tejido rizomático, que representa la interconexión con los otros sujetos que habitan el espacio virtual (Figura 3).

El videojuego desarrollado como parte de la narrativa transmedia fue denominado "Ball Connection" y se propuso como un juego estilo arcade, donde el jugador controla una esfera que deberá transitar por variadas situaciones y atravesar obstáculos para recuperar la conexión a Internet. Los escenarios buscan integrar las diferentes actividades y ámbitos sociales de la web. Cada nivel está asociado a un color y contienen diferentes elementos que identifican los distintos espacios representados: espacio Social, de Entretenimiento, de Trabajo, y un desafío final para recuperar la conexión (Figura 4).

\section{Caso 3: Grupo Brouet, Guberman, Malqui, Schneider, Stizza}

La propuesta de este grupo se centra en definir las interrelaciones posibles entre el espacio físico y el espacio personal en la web. Contextualizan su propuesta en un futuro no muy lejano, donde el personaje principal del video descubre sorpresivamente que distintos artefactos eléctricos sirven como "portales" o dispositivos input/output para acceder al espacio virtual.

Esta situación se representa en la escena del video en la que el personaje sube a un ascensor, ve un nuevo comando en el tablero y rápidamente lo presiona. De repente, se encuentra inmerso dentro de un espacio virtual donde hay elementos que no le son ajenos, ya que son sus propios recuerdos, historias y anécdotas almacenadas en la memoria RAM, representada a través de esferas paramétricas (Figura 5). Interactúa con su mundo virtual y termina por comprender el funcionamiento de las esferas y del espacio en sí. Minimiza su espacio, que le cabe en la palma de su mano, y vuelve al espacio físico donde su visión fue modificada, pudiendo observar una realidad aumentada a su alrededor.

La premisa del grupo fue proponer un espacio personal en la web como un ámbito no escindido y aislado, sino entrelazado con la vida cotidiana. Plantean la idea de la "expansión virtual", en la que el mismo usuario se "actualiza" en relación con el espacio y con otros usuarios.

Como parte de la narrativa, "VirtualMan" es la propuesta que realizaron como hipermedio lúdico, en donde el usuario tiene como objetivo llegar al ascensor o portal entre espacios, superando los diferentes obstáculos que se le presentan en los distintos escenarios (Figura 6).

\section{Discusión}

A fin de realizar un análisis comparativo de los resultados de la experiencia, se han definido las categorías a partir de los componentes que conforman el signo tríadico de Pierce y que son resignificados por Güerri (2014): Forma (atributos de diseño), Existencia (aspectos estructurales y tecnológicos), y Valor (contenidos y aspectos simbólicos). Éstos a su vez, se 
han dividido en sub-categorías que incorporan los Principios de Diseño y Evaluación de Espacios-Interfaz (Tosello, 2016) y la Teoría de las Inteligencias Múltiples (Gardner, 1999).

Las categorías y sub-categorías utilizadas para analizar cada caso son:

\section{FORMA:}

- Diseño: estética, tipo de contenido predominante, similitud con elementos del espacio físico, identidad (visibilidad, gama cromática, memorabilidad, percepción de la totalidad), consistencia, composición (estructuración del sitio), uso de metáforas, imágenes, códigos visuales y secuenciales, sonidos y elementos web.

\section{EXISTENCIA:}

- Tecnología: adaptabilidad (en cuáles soportes se puede visualizar y a través de qué tecnologías), recursos (tipo, cuestiones técnicas como peso, formato, cantidad de archivos, etc.).

- Comunicación, interacción, cooperación: vinculación entre medios disponibles, incorporación de redes sociales (Facebook, Twitter, mail, etc.), sistema de integración de aportes/colaboraciones, posibilidad de establecer espacios de trabajo individuales y colectivos, sistema de visualización de aportes, herramientas de evaluación y visualización de interacción, rol del usuario (activo/pasivo) y permisos, tipo y grado de participación (sincrónica, asincrónica, individual, grupal), nivel de interactividad y cooperación entre usuarios, posibilidad de personalización.

- Heurística, usabilidad: estructura de navegación, posibilidades de recorrido, usabilidad (comprensión, uso de objetos estándar, lenguaje de los usuarios, facilidad de aprendizaje, simplicidad, eficiencia, estado y seguimiento de las acciones, encontrabilidad, visibilidad del estado del sistema, funcionalidad, estructuración de contenidos, sistema de búsqueda, cooperación de la estética en la usabilidad, facilidad de recordación, ayuda brindada), y accesibilidad.

\section{VALOR:}

- Temática: argumento, originalidad de los contenidos, identificación del objetivo de la propuesta, consistencia del relato en los distintos medios narrativos, modos de representación.
- Inteligencias múltiples: tipos de inteligencias que contempla y/o estimula la narrativa propuesta:

- Lingüística: presencia de texto oral o escrito, uso del lenguaje.

- Musical: presencia de sonido, voz humana, ruidos y efectos sonoros, música.

- Lógico - matemática: uso de operaciones básicas, posibilidad de establecer deducciones, resolución de problemas con o sin tiempo.

- Espacial: representación 2D y 3D, uso de mapas, diagramas, utilización de instancias de un mismo elemento.

- Cinético-corporal: requerimientos de movimientos corporales por parte del usuario, movimientos funcionales, movimientos expresivos, manejo de objetos.

- Intrapersonal e Interpersonal: posibilidad de actividades individuales, actividades grupales, actividades colectivas.

- Naturalista: interacción con seres vivos, reconocimiento del entorno.

A partir de estas categorías y sub-categorías, fue posible analizar los casos considerando las potencialidades y limitaciones que presentan en relación a la temática abordada, y fundamentalmente, sus diferentes estrategias de abordaje.

En la propuesta del Caso 1, se destacan claramente los valores de la Forma por encima de los aspectos tecnológicos o narrativos, que si bien son parte integrante de la propuesta, han sido desarrollados con menor profundidad. El elaborado diseño de los escenarios, el gran nivel de detalle con que fueron ideados los personajes, el uso de una única gama cromática, la utilización de metáforas, códigos visuales, sonidos y lenguajes propios, así como la espacialidad lograda, otorgan una clara identidad estética al espacio personal propuesto.

Por su parte el Caso 2, en la estrategia de abordaje del problema, se han priorizado los aspectos tecnológicos y de interacción vinculados a la Existencia. Este grupo optó por
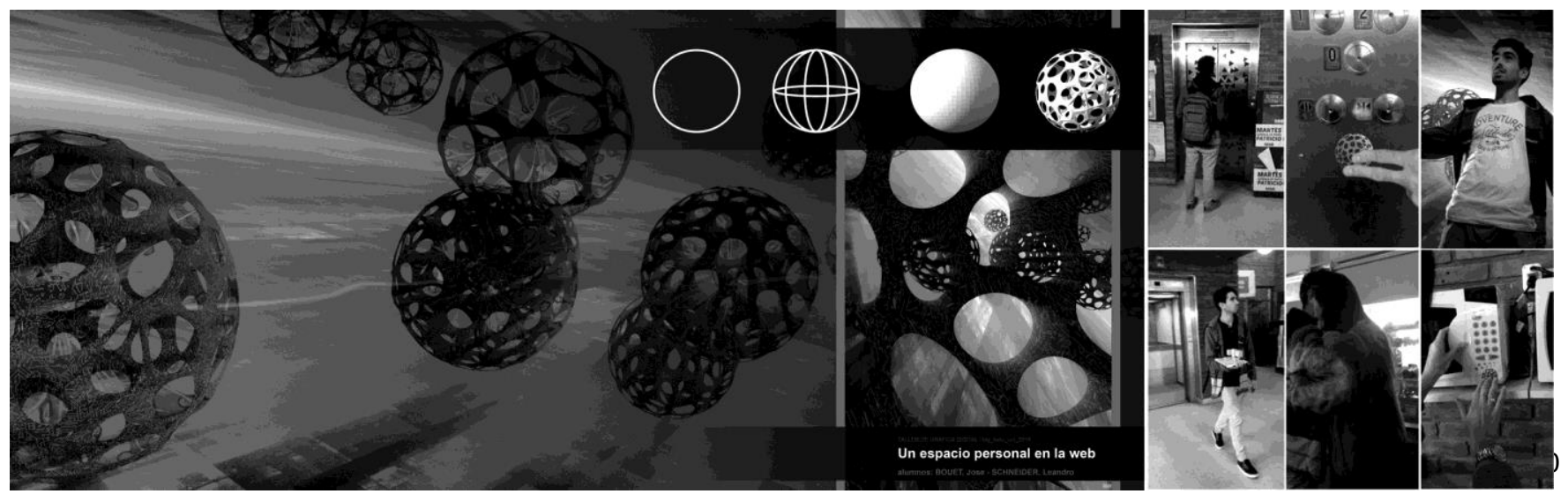

Figura 5: Modelo 3D y escenas video digital Caso 3 (Grupo Brouet, Guberman, Malqui, Schneider, Stizza). 

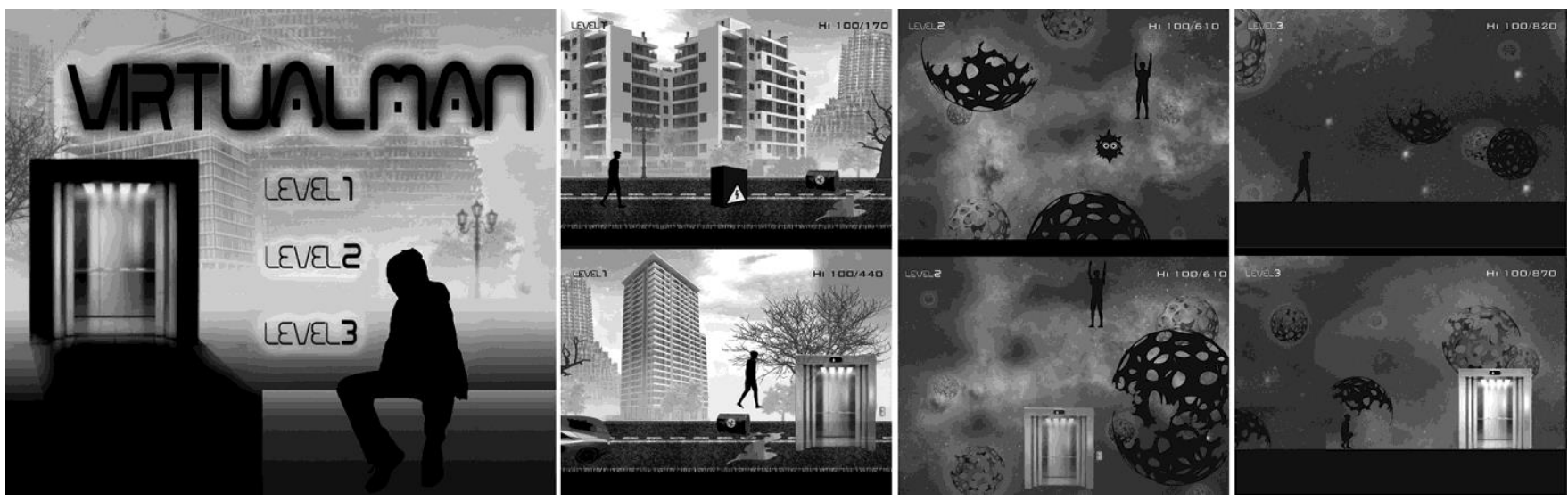

Figura 6: Hipermedio Lúdico Caso 3 (Grupo Brouet, Guberman, Malqui, Schneider, Stizza).

una estética geométrica y abstracta, enfocándose en resolver aspectos técnicos vinculados a la integración de las redes sociales, medios y actividades disponibles, en el diseño de distintos ámbitos virtuales de interacción social, trabajo y entretenimiento, previendo distintos grados de participación individual y grupal.

En tanto en los Casos 1 y 2 se proponen espacios personales que se limitan exclusivamente al ámbito virtual, en el Caso 3 se desarrolla una propuesta de interacción e interrelación entre espacios físicos y virtuales, resaltándose aquí los aspectos vinculados al Valor de los contenidos y la originalidad del argumento. Sin embargo, al contextualizarse la idea en el futuro, los aspectos tecnológicos posibilitantes del espacio virtual planteado, fueron resueltos con cierto grado de indefinición, de la misma manera que la estética propuesta para ese espacio personal inmersivo.

Siguiendo los objetivos del Taller de Grafica Digital, que en tanto actividad académica promueve reflexionar sobre la tarea del diseñador como sujeto potenciador de respuestas innovadoras y creativas en relación a un nuevo paradigma epistemológico, estético y técnico, cabe destacar el valor de todas las propuestas que desde diferentes abordajes, han experimentado estrategias novedosas de diseño y representación, integrando las potencialidades del medio digital y asumiendo una actitud participativa, exploratoria y lúdica.

El debate acerca de la habitabilidad físico-virtual continúa abierto, y uno de los tantos desafíos que deben afrontar el diseño y la arquitectura sigue siendo explorar, imaginar y construir nuevos espacios habitables y posibles escenarios en la "nube" que expresen e integren múltiples formas de habitar.

\section{Agradecimientos}

Agradecemos a Matías Dalla Costa, Álvaro Dorigo y Cecilia Zorzón; y a la Universidad Nacional del Litoral por subsidiar el proyecto "Diseño, desarrollo y evaluación de espaciosinterfaz destinados a actividades de docencia, investigación y extensión en la universidad pública argentina".

\section{Referencias}

Bachelard, G. (1975). La poética del espacio. Madrid: Fondo de Cultura Económica.

Bahamondes, F., Fredes, C. (2014). Principios de diseño de juegos ubicuos. SIGraDi Design in Freedom. Montevideo: Universidad de la República. Recuperado de: http://pdf.blucher.com.br.s3-saeast-1.amazonaws.com/designproceedings/sigradi2014/0091.pdf

Cátedra Latinoamericana de Narrativas Transmedia. Recuperado de: http://catedratransmedia.com.ar/desiguales-documentaltransmedia/

Flusser, V. (1967). Jogos. Recuperado de: http://geccom.incubadora.fapesp.br/portal/referencias/textos/flus ser/jogos.pdf

Foucault, M. (1994). Espacios Diferentes. En Toponimias, Ocho ideas del espacio. Madrid: La Caixa.

García Canal, M. I. (1999). Foucault, filósofo del espacio. http://es.scribd.com/doc/60908757/Foucault-filosofo-del-espacio

Gardner, H. (1999). La inteligencia reformulada. Las Inteligencias Múltiples en el Siglo XXI. Barcelona, Paidós.

Guerri, C. (2014). Nonágono Semiótico un modelo operativo para la investigación cualitativa. Buenos Aires: Eudeba.

Hall, E. (1966). The Hidden Dimension. Garden City, N.Y.: Doubleday.

Holahan, Ch. J. (2000). Psicología Ambiental. Un enfoque general. México: Limusa.

Jenkins, H. (2009). The Revenge of the Origami Unicorn: Seven Principles of Transmedia Storytelling. Recuperado de: http://henryjenkins.org/2009/12/the revenge of the origami uni. $\underline{\mathrm{html}}$

Merleau-Ponty, M. (1957). La fenomenología de la percepción. Madrid: Fondo de Cultura Económica.

Norberg Schulz, C. (1975). Existencia, espacio y arquitectura. Barcelona: Blume.

Scolari, C. (2013). Narrativas transmedia. Cuando todos los medios cuentan. Barcelona: Deusto.

Sommer, R. (1969). Personal space: the behavior basis of design. New Jersey: Prentice-Hall.

Tosello, M. (2016). El espacio-interfaz del dispositivo hipermedial dinámico. Tesis doctoral. Universidad Nacional de Rosario. 